\title{
Long-time behavior of solutions to Hamilton-Jacobi equations with quadratic gradient term
}

\author{
Yasuhiro Fujita and Paola Loreti
}

\begin{abstract}
We study a rate of convergence appearing in the long-time behavior of viscosity solutions of the Cauchy problem for the Hamilton-Jacobi equation$$
u_{t}(x, t)+\alpha x \cdot D u(x, t)+\beta|D u(x, t)|^{2}=f(x) \quad \text { in } \mathbb{R}^{n} \times(0, \infty),
$$

where $\alpha, \beta>0$ are constants and $f$ is a Lipschitz and semiconvex function on $\mathbb{R}^{n}$. Our goal of this paper is to show that the semiconvexity property of $f$ is an important factor which determines this rate of convergence. We also establish existence, uniqueness and Lipschitz continuity of viscosity solutions of the Cauchy problem and the corresponding ergodic problem for HamiltonJacobi equations in $\mathbb{R}^{n}$.
\end{abstract}

Mathematics Subject Classification (2000). 35K55, 35B40, 49L25.

Keywords. Rates of convergence, Hamilton-Jacobi equation, Viscosity solutions, Semiconvexity.

\section{Introduction}

Recently, the long-time asymptotics of viscous Hamilton-Jacobi equations and Hamilton-Jacobi equations have been investigated by many authors. We refer to $[2-4,6,7,14,16,17]$ for asymptotic results in compact domains. Motivated by these development, the authors $[9,10]$ have recently investigated the asymptotic problem for viscous Hamiltonian-Jacobi equations with the Ornstein-Uhlenbeck operator

$$
u_{t}(x, t)-\Delta u(x, t)+\alpha x \cdot D u(x, t)+H(D u(x, t))=f(x) \text { in } \mathbb{R}^{n} \times(0, \infty),
$$
JSPS. 
and the corresponding Hamiltonian-Jacobi equations

$$
u_{t}(x, t)+\alpha x \cdot D u(x, t)+H(D u(x, t))=f(x) \text { in } \mathbb{R}^{n} \times(0, \infty),
$$

where $H$ is a convex function on $\mathbb{R}^{n}, \Delta$ denotes the Laplace operator, and $\alpha$ is a positive constant, and has established a convergence result similar to those obtained by $[2,6,7,17]$. The result for $(1.1)$ states that there are a constant $c \in \mathbb{R}$ and a viscosity solution $v$ of the Hamilton-Jacobi equation of ergodic type

$$
c+\alpha x \cdot D v(x)+H(D v(x))=f(x) \text { in } \mathbb{R}^{n}
$$

such that

$$
\lim _{t \rightarrow \infty}(u(\cdot, t)-c t)=v \text { locally uniformly in } \mathbb{R}^{n} .
$$

In this paper, we are mainly concerned about a rate of convergence in (1.3). To begin our investigation, we consider a simplified problem

$$
\begin{array}{ll}
u_{t}(x, t)+\alpha x \cdot D u(x, t)+\beta|D u(x, t)|^{2}=f(x) & \text { in } \mathbb{R}^{n} \times(0, \infty), \\
u(\cdot, 0)=u_{0} & \text { in } \mathbb{R}^{n}
\end{array}
$$

where $u: \mathbb{R}^{n} \times[0, \infty) \mapsto \mathbb{R}$ is the unknown function, $u_{t}=\partial u / \partial t, D u$ denotes its gradient, $\alpha, \beta>0$ are constants, and $f$ and $u_{0}$ are given Lipschitz continuous functions on $\mathbb{R}^{n}$. In this simpler case, we are able to clarify a factor which determines the rate of convergence in (1.3). Furthermore, we give examples which show that our result is optimal.

In this case, the corresponding Hamilton-Jacobi equation is given by

$$
c+\alpha x \cdot D v(x)+\beta|D v(x)|^{2}=f(x) \text { in } \mathbb{R}^{n} .
$$

An important assumption is that $f$ and $u_{0}$ are Lipschitz continuous on $\mathbb{R}^{n}$. By [10], there is a constant $c \in \mathbb{R}$ and a viscosity solution $v \in C\left(\mathbb{R}^{n}\right)$ of (1.6) such that (1.3) holds.

Let us recall that a function $g \in C\left(\mathbb{R}^{n}\right)$ is said to be semiconvex on $\mathbb{R}^{n}$, if $g(x)+\mu|x|^{2}$ is convex on $\mathbb{R}^{n}$ for some constant $\mu>0$. When $g \in \operatorname{Lip}\left(\mathbb{R}^{n}\right)$ is semiconvex on $\mathbb{R}^{n}$, we define the constant $I(g)$ by

$$
I(g)=\inf \left\{\mu \mid \mu>0 \text { and } g(x)+\mu|x|^{2} \text { is convex on } \mathbb{R}^{n}\right\} .
$$

Here, by $\operatorname{Lip}\left(\mathbb{R}^{n}\right)$, we denote the set of Lipschitz continuous functions on $\mathbb{R}^{n}$. Note that if $g$ itself is convex on $\mathbb{R}^{n}$, we see that $I(g)=0$.

Our result is that if $f$ is Lipschitz continuous and semiconvex on $\mathbb{R}^{n}$ with $I(f) \in\left[0, \alpha^{2} / 4 \beta\right)$, then, for any $u_{0} \in \operatorname{Lip}\left(\mathbb{R}^{n}\right)$, there is a constant $C_{1}>0$ depending on the Lipschitz constants of $f$ and $u_{0}$ such that

$$
|u(x, t)-(c t+v(x))| \leq \frac{C_{1}}{\theta} e^{-\theta t}(1+|x|) \quad \text { in } \mathbb{R}^{n} \times[0, \infty),
$$

where $\theta=\sqrt{\alpha^{2}-4 \beta I(f)}$. We observe that $\theta$ depends on only $I(f)$, and is independent of the Lipschitz constants of $f$ and $u_{0}$. 
Furthermore, for any constant $I_{0} \in\left[0, \alpha^{2} / 4 \beta\right)$, we construct $f, u_{0} \in \operatorname{Lip}\left(\mathbb{R}^{n}\right)$ such that $I(f)=I_{0}$ and

$$
\lim _{t \rightarrow \infty} e^{t \sqrt{\alpha^{2}-4 \beta I_{0}}}[u(x, t)-(c t+v(x))]=-\delta|x| \quad \text { for }|x| \leq 1,
$$

where $\delta>0$ is a constant, which shows the optimality. Also we can not enlarge the interval $\left[0, \alpha^{2} / 4 \beta\right)$, because if $I(f)=\alpha^{2} / 4 \beta$ then we can construct, as a special case of [11], an example in which the rate of convergence in (1.3) is not necessarily of exponential order, that is more precisely

$$
\lim _{t \rightarrow \infty} t[u(x, t)-(c t+v(x))]=\frac{1}{4 \beta}|x|^{2} \quad \text { for }|x| \leq 1 .
$$

By (1.8) and these two examples, we see that the semiconvexity of $f$ is an important factor which determines the rate of convergence in (1.3). Furthermore, the constant $I(f)$ is a good parameter to describe this rate. However, for a general Hamilton-Jacobi equation (1.1), we can not find a factor which determines the rate of convergence in (1.3).

The contents of this paper are as follows: In Sect. 2, we state existence, uniqueness and Lipschitz continuity of viscosity solutions of the Cauchy problem (1.1) and (1.5). In Sect. 3, we show that there is a viscosity solution $v \in \operatorname{Lip}\left(\mathbb{R}^{n}\right)$ of (1.2) for a constant $c \in \mathbb{R}$. In Sect. 4 , we derive the estimate (1.8) for a unique viscosity solution of the Cauchy problem (1.4) and (1.5). We also give two examples which clarify the factor which determines the rate of convergence in (1.3). In Sect. 5, we prove some results of Sect. 2 .

\section{Cauchy problem for Hamilton-Jacobi equation}

In this section, we state existence, uniqueness and Lipschitz estimates of viscosity solutions of (1.1) and (1.5). The results of this section and Sect. 3 are mainly due to personal communications with Professor Hitoshi Ishii [13]. We give a modification of his results. The new results of this section, with respect to previous joint works (see $[9,10]$ ) are Lipschitz estimates of viscosity solutions of (1.1) and (1.5). We give their proofs in Sect. 5 below.

We consider the following more general Hamilton-Jacobi equation:

$$
u_{t}(x, t)-\sigma \Delta u(x, t)+\alpha x \cdot D u(x, t)+H(D u(x, t))=f(x) \text { in } \mathbb{R}^{n} \times(0, \infty),
$$

where $0 \leq \sigma \leq 1$. We assume the following:

$$
\begin{aligned}
& H \in \operatorname{Lip}_{l o c}\left(\mathbb{R}^{n}\right), \\
& \alpha>0 \text { is a constant, and } f \in \operatorname{Lip}\left(\mathbb{R}^{n}\right), \\
& u_{0} \in \operatorname{Lip}\left(\mathbb{R}^{n}\right) .
\end{aligned}
$$

The Hamilton-Jacobi equation (1.4) is the case such that $\sigma=0$ and $H(p)=\beta|p|^{2}$. Let us denote by $L_{f}$ and $L_{u_{0}}$ the Lipschitz constants of $f$ and $u_{0}$, respectively. 
Define the constant $M$ by

$$
M=\max \left\{L_{u_{0}}, \frac{L_{f}}{\alpha}\right\} .
$$

Let

$$
Q=\mathbb{R}^{n} \times(0, \infty), \quad B(a, R)=\left\{x \in \mathbb{R}^{n}|| x-a \mid \leq R\right\} \quad \text { for } a \in \mathbb{R}^{n}, R>0 .
$$

Proposition 2.1. Assume (2.2)-(2.4). For $0 \leq \sigma \leq 1$, let $u \in U S C(Q)$, $v \in$ $L S C(Q)$ be a viscosity subsolution and a viscosity supersolution of the HamiltonJacobi equation (2.1), respectively. Assume that for each $T>0$ there is a constant $C_{T}>0$ such that

$$
u(x, t) \vee(-v(x, t)) \leq C_{T}(1+|x|) \quad \text { in } \mathbb{R}^{n} \times(0, T),
$$

and that for each $R>0$,

$$
\lim _{\epsilon \searrow 0} \sup \left\{\left(u(x, t)-u_{0}(x)\right) \vee\left(u_{0}(x)-v(x, t)\right) \mid t \in(0, \epsilon), x \in B(0, R)\right\} \leq 0 .
$$

Then, $u(x, t)-v(y, t) \leq M|x-y|$ for all $(x, t),(y, t) \in \bar{Q}$.

Proposition 2.2. Assume (2.2)-(2.4). Let $0 \leq \sigma \leq 1$. Then, the Cauchy problem (2.1) and (1.5) admits a unique viscosity solution $u^{\sigma} \in C(\bar{Q})$ such that

$$
\begin{aligned}
\left|u^{\sigma}(x, t)-u^{\sigma}(y, t)\right| & \leq M|x-y| \quad \text { for }(x, t),(y, t) \in \bar{Q}, \\
\left|u^{\sigma}(x, t)-u^{\sigma}(x, s)\right| & \leq \omega_{\sigma, R}(|t-s|) \quad \text { for } x \in B(0, R), t, s \in[0, \infty), R>0,
\end{aligned}
$$

where

$$
\omega_{\sigma, R}(t)=A[\sqrt{t \sigma(1+\alpha t)}+t(1+R)]
$$

for some constant $A>0$ which is independent of $R, \sigma$ and $t$.

Remark 2.3. When $0<\sigma \leq 1$, Propositions 2.1 and 2.2 were shown in [9]. When $\sigma=0$, existence and uniqueness of viscosity solutions of (2.1) and (1.5) was shown in a class $C\left(\mathbb{R}^{n} \times[0, \infty)\right)$ in $[10]$.

Remark 2.4. Note that $u^{0} \in \operatorname{Lip}(\bar{Q})$. When $\sigma>0$, we have $u^{\sigma} \in C^{2,1}(Q) \cap C(\bar{Q})$ by $[9]$.

Proposition 2.5. Assume (2.2)-(2.4). For $0<\sigma \leq 1$, let $u^{\sigma} \in C^{2,1}(Q) \cap C(\bar{Q})$ be a unique solution of (2.1) and (1.5). Then, there is a sequence $\left\{\sigma_{j}\right\}$ such that $\sigma_{j} \searrow 0$ and

$$
u^{\sigma_{j}} \rightarrow u \text { locally uniformly on } \bar{Q},
$$

where $u$ is a unique viscosity solution of (2.1) and (1.5) for $\sigma=0$. 
Proof. Let $\omega_{\sigma, R}$ be the modulus of continuity of $u^{\sigma}$ given by (2.10). We have $\omega_{\sigma, R}(\cdot) \leq \omega_{1, R}(\cdot)$ on $[0, \infty)$ for each $0<\sigma \leq 1$. Hence, by (2.8)-(2.9), we have

$$
\begin{aligned}
& \left|u^{\sigma}(x, t)-u^{\sigma}(y, s)\right| \leq\left|u^{\sigma}(x, t)-u^{\sigma}(y, t)\right|+\left|u^{\sigma}(y, t)-u^{\sigma}(y, s)\right| \\
& \quad \leq M|x-y|+\omega_{1, R}(|t-s|) \text { for } x, y \in B(0, R), t, s \in[0, \infty), R>0 .
\end{aligned}
$$

Thus, the set $\left\{u^{\sigma} \mid 0<\sigma \leq 1\right\}$ is equi-continuous on compact sets of $\bar{Q}$. In particular, putting $y=0$ and $s=0$ in (2.12), we have

$$
\left|u^{\sigma}(x, t)\right| \leq\left|u_{0}(0)\right|+M|x|+\omega_{1, R}(t) \text { for } x \in B(0, R), t \in[0, \infty), R>0 .
$$

Thus, the set $\left\{u^{\sigma} \mid 0<\sigma \leq 1\right\}$ is equi-bounded on compact sets of $\bar{Q}$. Using the Ascoli-Arzelá theorem and a uniqueness result in Proposition 2.1, we obtain (2.11) by a stability result for viscosity solutions (cf. [5]).

\section{Ergodic problem for Hamilton-Jacobi equation}

In this section, we consider existence and Lipschitz continuity of solutions to the ergodic problem (1.2). In (1.2), the solution to be found is a pair $(c, v) \in$ $\mathbb{R} \times \operatorname{Lip}\left(\mathbb{R}^{n}\right)$. We consider the following more general ergodic problem:

$$
c-\sigma \Delta v(x)+\alpha x \cdot D v(x)+H(D v(x))=f(x) \text { in } \mathbb{R}^{n},
$$

where $0 \leq \sigma \leq 1$. Here, we assume (2.2) and (2.3). The ergodic problem (1.6) is the case such that $\sigma=0$ and $H(p)=\beta|p|^{2}$. Let

$$
C_{f}=\sup \left\{\frac{|f(x)|}{\left(1+|x|^{2}\right)^{1 / 2}} \mid \quad x \in \mathbb{R}^{n}\right\} .
$$

Proposition 3.1. Assume (2.2) and (2.3). Let $0 \leq \sigma \leq 1$. Then, there exists a constant $c^{\sigma} \in \mathbb{R}$ such that (3.1) has a viscosity solution $v^{\sigma} \in \operatorname{Lip}\left(\mathbb{R}^{n}\right)$ satisfying

$$
\begin{aligned}
\left|v^{\sigma}(x)-v^{\sigma}(y)\right| & \leq \frac{L_{f}}{\alpha}|x-y| \text { for } x, y \in \mathbb{R}^{n}, \\
\left|c^{\sigma}\right| & \leq \frac{C_{f}}{\alpha}(n+\alpha)+\max \left\{|H(p)||| p \mid \leq \frac{C_{f}}{\alpha}\right\} .
\end{aligned}
$$

The main observation toward proving Proposition 3.1 is a comparison theorem for the following discounted control problem:

$$
\lambda v_{\lambda}(x)-\sigma \Delta v_{\lambda}(x)+\alpha x \cdot D v_{\lambda}(x)+H\left(D v_{\lambda}(x)\right)=f(x) \text { in } \mathbb{R}^{n},
$$

where $0<\lambda<1$ is a constant.

Proposition 3.2. Assume (2.2) and (2.3). For $0 \leq \sigma \leq 1$ and $0<\lambda<1$, let $u \in U S C\left(\mathbb{R}^{n}\right), v \in L S C\left(\mathbb{R}^{n}\right)$ be a viscosity subsolution and a viscosity supersolution of (3.4), respectively. Assume that there is a constant $C>0$ such that

$$
u(x) \vee(-v(x)) \leq C(1+|x|) \text { for } x \in \mathbb{R}^{n} .
$$

Then

$$
u(x)-v(y) \leq \frac{L_{f}}{\alpha}|x-y| \text { for } x, y \in \mathbb{R}^{n}
$$


The proof of Proposition 3.2 is similar to that of Proposition 2.1. So, we omit it (for the proof of Proposition 2.1, see Sect. 5).

Proof of Proposition 3.1. 1. Define the function $U^{\lambda, \sigma}$ on $\mathbb{R}^{n}$ by $U^{\lambda, \sigma}(x)=\frac{C_{f}}{\lambda+\alpha}\left(1+|x|^{2}\right)^{1 / 2}+\frac{1}{\lambda}\left[\frac{C_{f}}{\lambda+\alpha}(n+\alpha)+\max \left\{|H(p)||| p \mid \leq \frac{C_{f}}{\lambda+\alpha}\right\}\right]$.

Then, it is easy to see that $U^{\lambda, \sigma}$ and $-U^{\lambda, \sigma}$ are, respectively, a viscosity supersolution and a subsolution of (3.4). Hence, the Perron method gives a viscosity solution $v^{\lambda}$ of (3.4) defined by

$$
v^{\lambda, \sigma}(x)=\inf \left\{w(x) \mid-U^{\lambda, \sigma} \leq w \leq U^{\lambda, \sigma} \text { in } \mathbb{R}^{n}, w\right. \text { is a supersolution of (3.4)\}. }
$$

By Proposition 3.2, $v^{\lambda, \sigma}$ satisfies

$$
\left|v^{\lambda, \sigma}(x)-v^{\lambda, \sigma}(y)\right| \leq \frac{L_{f}}{\alpha}|x-y| \text { for } x, y \in \mathbb{R}^{n} .
$$

Hence, $v^{\lambda, \sigma}$ is a Lipschitz continuous viscosity solution of (3.4).

2. Let

$$
w^{\lambda, \sigma}(x)=v^{\lambda, \sigma}(x)-v^{\lambda, \sigma}(0), \quad x \in \mathbb{R}^{n} .
$$

Then, we have

$$
\begin{gathered}
\left|w^{\lambda, \sigma}(x)\right| \leq \frac{L_{f}}{\alpha}|x| \text { for } x \in \mathbb{R}^{n} \\
\left|w^{\lambda, \sigma}(x)-w^{\lambda, \sigma}(y)\right| \leq \frac{L_{f}}{\alpha}|x-y| \text { for } x, y \in \mathbb{R}^{n} .
\end{gathered}
$$

Hence, the set $\left\{w^{\lambda, \sigma} \mid 0<\lambda<1\right\}$ is equi-bounded and equi-continuous on compact sets of $\mathbb{R}^{n}$. Furthermore, by (3.7), we have

$$
\lambda\left|v^{\lambda, \sigma}(0)\right| \leq \frac{\lambda C_{f}}{\alpha}+\frac{C_{f}}{\alpha}(n+\alpha)+\max \left\{|H(p)||| p \mid \leq \frac{C_{f}}{\alpha}\right\} \text { for } 0<\lambda<1 .
$$

By the Ascoli-Arzelá theorem, we find a sequence $\left\{\lambda_{j}\right\}$ and a pair $\left(c^{\sigma}, v^{\sigma}\right) \in$ $\mathbb{R} \times C\left(\mathbb{R}^{n}\right)$ such that $\lambda_{j} \searrow 0$ as $j \rightarrow \infty$ and

$$
\begin{aligned}
& \lambda_{j} v_{\lambda_{j}, \sigma}(0) \rightarrow c^{\sigma}, \\
& w_{\lambda_{j}, \sigma} \rightarrow v^{\sigma} \text { locally uniformly on } \mathbb{R}^{n} .
\end{aligned}
$$

For this constant $c^{\sigma}$, the function $v^{\sigma}$ is a viscosity solution to (3.1) with (3.2) by a stability result for viscosity solutions (cf. [5]). It is easy to see (3.3). The proof is complete

Remark 3.3. [9] When $0<\sigma \leq 1$, we have $v^{\sigma} \in C^{2}\left(\mathbb{R}^{n}\right)$. Similarly, when $\lambda>0$ and $0<\sigma \leq 1$, we have $v^{\lambda, \sigma} \in C^{2}\left(\mathbb{R}^{n}\right)$. Furthermore, for $0<\sigma \leq 1$, the following uniqueness result holds: if $v_{j}^{\sigma} \in \operatorname{Lip}\left(\mathbb{R}^{n}\right)$ is a viscosity solution to (3.1) for a constant $c_{j}^{\sigma} \in \mathbb{R}(j=1,2)$, then $c_{1}^{\sigma}=c_{2}^{\sigma}$ and $v_{1}^{\sigma}-v_{2}^{\sigma}=$ a constant in $\mathbb{R}^{n}$. 
Finally, we state a stability result for $\left\{\left(c^{\sigma}, v^{\sigma}\right) \mid 0<\sigma \leq 1\right\}$. The following proposition follows from (3.2) and (3.3), the Ascoli-Arzelá theorem and a stability result for viscosity solutions (cf. [5]).

Proposition 3.4. Assume (2.2) and (2.3). For $0<\sigma \leq 1$, let $v^{\sigma} \in C^{2}\left(\mathbb{R}^{n}\right) \cap$ $\operatorname{Lip}\left(\mathbb{R}^{n}\right)$ be a solution of (3.1) for a constant $c^{\sigma} \in \mathbb{R}$. Then, there is a sequence $\left\{\sigma_{j}\right\}$ such that $\sigma_{j} \searrow 0$ and

$$
c^{\sigma_{j}} \rightarrow c, \quad v^{\sigma_{j}} \rightarrow v \text { locally uniformly on } \mathbb{R}^{n},
$$

where $v \in \operatorname{Lip}\left(\mathbb{R}^{n}\right)$ is s viscosity solution of (3.1) for $\sigma=0$ and the constant $c$.

Remark 3.5. Assume (2.2) and (2.3). Let

$$
c_{0}=\min \left\{f(x)+\frac{\alpha^{2}}{4 \beta}|x|^{2} \mid x \in \mathbb{R}^{n}\right\} .
$$

Then, $c_{0}$ is a unique constant such that (3.1) for $\sigma=0$ admits a viscosity solution $v \in \operatorname{Lip}\left(\mathbb{R}^{n}\right)[12]$. Hence, the constant $c$ in Proposition 3.4 coincides with $c_{0}$.

\section{Rate of convergence}

In this section, we derive the estimate (1.8) for a unique viscosity solution $u \in$ $\operatorname{Lip}(\bar{Q})$ of the Cauchy problem (1.4) and (1.5). We also give two examples to clarify the factor which determines the rate of convergence in (1.3). In the following, we denote by $u$ a unique viscosity solution in $\operatorname{Lip}(\bar{Q})$ of (1.4) and (1.5). We assume:

$$
\beta>0 \text {, and } f(x) \text { is semiconvex on } \mathbb{R}^{n} .
$$

Let $I(f)$ be the constant in (1.7).

Theorem 4.1. Assume (2.3) and (4.1). If $I(f)<\alpha^{2} / 4 \beta$, then there is a constant $c \in \mathbb{R}$ such that (1.6) has a viscosity solution $v \in \operatorname{Lip}\left(\mathbb{R}^{n}\right)$ satisfying the following:

(i) $v(x)+K|x|^{2}$ is convex on $\mathbb{R}^{n}$, where

$$
K=\frac{\alpha-\theta}{4 \beta}, \quad \theta=\sqrt{\alpha^{2}-4 \beta I(f)} .
$$

(ii) For any $u_{0} \in \operatorname{Lip}\left(\mathbb{R}^{n}\right)$, we have

$$
|u(x, t)-(c t+v(x))| \leq \frac{2 M}{\theta} e^{-\theta t}(\alpha|x|+2 \beta M) \quad \text { in } \bar{Q},
$$

where $M$ is the constant of (2.5).

Remark 4.2. Note that, in Theorem 4.1(ii), the constant $\theta$ is independent of the Lipschitz constant of $f$ and $u_{0}$; it depends on only $I(f)$. If $f \in \operatorname{Lip}\left(\mathbb{R}^{n}\right)$ is convex on $\mathbb{R}^{n}$, then we have $\theta=\alpha$. 
Remark 4.3. Let $f \in \operatorname{Lip}\left(\mathbb{R}^{n}\right)$. Then, the Aubry set $\mathcal{A}$ of the Hamilton-Jacobi equation (1.4) is given by

$$
\mathcal{A}=\left\{\left.x \in \mathbb{R}^{n}\left|f(x)+\frac{\alpha^{2}}{4 \beta}\right| x\right|^{2}=c_{0}\right\}
$$

where $c_{0} \in \mathbb{R}$ is the constant of Remark $3.5[12]$. Hence, if $I(f)<\alpha^{2} / 4 \beta$, then $\mathcal{A}$ consists of a single point.

To show Theorem 4.1, we give two lemmas. Let $h \in \operatorname{LSC}\left(\mathbb{R}^{n}\right)$ be superlinear, i.e., $\lim _{|x| \rightarrow \infty} h(x) /|x|=\infty$. Define the convex envelope $h_{* *}$ of $h$ by

$h_{* *}(x)=\inf \left\{\sum_{j=1}^{k} \gamma_{j} h\left(x_{j}\right) \mid x=\sum_{j=1}^{k} \gamma_{j} x_{j}, \sum_{j=1}^{k} \gamma_{j}=1, \gamma_{j}>0, x_{j} \in \mathbb{R}^{n}, k \leq n+1\right\}$.

By [15, Corollary 17.1.5, p. 157], $h_{* *}$ is the largest convex function majorized by $h$ on $\mathbb{R}^{n}$.

Lemma 4.4. Let $g \in \operatorname{LSC}\left(\mathbb{R}^{n}\right)$. We assume that, for some constant $A>0, g$ verifies

$$
-A(1+|x|) \leq g(x) \quad \text { for } x \in \mathbb{R}^{n} .
$$

Define the function $h$ by $h(x)=g(x)+a|x|^{2}$ for a constant $a>0$. Then, there is a constant $C>0$ such that

$$
-\left(h_{* *}(x)-a|x|^{2}\right) \leq C(1+|x|) \quad \text { for } x \in \mathbb{R}^{n} .
$$

Proof. Let us define the function $V$ by

$$
V(x)=\left(|x|-\frac{A}{2 a}\right)^{2} \quad \text { for } x \in \mathbb{R}^{n} .
$$

It is easy to see that

$$
V_{* *}(x)=\chi_{\{|x| \geq A /(2 a)\}}(x)\left(|x|-\frac{A}{2 a}\right)^{2} \text { for } x \in \mathbb{R}^{n},
$$

where $\chi_{E}(x)$ is the characteristic function of a set $E$. In the following of this proof, the infimum is always taken over all $\left\{\left(x_{j}, \gamma_{j}\right)\right\}_{j=1}^{k}$ satisfying $x=\sum_{j=1}^{k} \gamma_{j} x_{j}$, $\sum_{j=1}^{k} \gamma_{j}=1, \gamma_{j}>0, x_{j} \in \mathbb{R}^{n}$ and $k \leq n+1$. Then, we have 


$$
\begin{aligned}
h_{* *}(x) & =\inf \left\{\sum_{j=1}^{k} \gamma_{j} h\left(x_{j}\right)\right\} \geq \inf \left\{\sum_{j=1}^{k} \gamma_{j}\left[-A\left(1+\left|x_{j}\right|\right)+a\left|x_{j}\right|^{2}\right]\right\} \\
& =-A+\inf \left\{\sum_{j=1}^{k} \gamma_{j}\left[a\left(\left|x_{j}\right|-\frac{A}{2 a}\right)^{2}-\frac{A^{2}}{4 a}\right]\right\} \\
& =-A-\frac{A^{2}}{4 a}+a \inf \left\{\sum_{j=1}^{k} \gamma_{j}\left(\left|x_{j}\right|-\frac{A}{2 a}\right)^{2}\right\} \\
& =-A-\frac{A^{2}}{4 a}+a V_{* *}(x) .
\end{aligned}
$$

By (4.4), it is easy to see that there is a constant $C^{*}>0$ such that

$$
V_{* *}(x) \geq|x|^{2}-C^{*}(1+|x|) \text { for } x \in \mathbb{R}^{n} .
$$

The proof is complete.

In the following, for $0<\sigma<1$ and $u_{0} \in \operatorname{Lip}\left(\mathbb{R}^{n}\right)$, we denote by $u^{\sigma} \in$ $C^{2,1}(Q) \bigcap C(\bar{Q})$ a unique solution of (1.4) and (1.5) given by Proposition 2.2 and Remark 2.4. In the same way, we denote by $\left(c^{\sigma}, v^{\sigma}\right) \in \mathbb{R} \times C^{2}\left(\mathbb{R}^{n}\right)$ a unique solution of (1.6) given by Proposition 3.1 and Remark 3.3. Here, uniqueness of $v^{\sigma}$ is up to an additive constant.

Lemma 4.5. Assume (2.3) and (4.1). Then, for each $0<\sigma<1$,

$$
v^{\sigma}(x)+K|x|^{2} \text { is convex on } \mathbb{R}^{n} \text {. }
$$

Furthermore, for $u_{0} \in \operatorname{Lip}\left(\mathbb{R}^{n}\right)$, the function

$$
\xi^{\sigma}(x, t):=u^{\sigma}(x, t)-\left(c^{\sigma} t+v^{\sigma}(x)\right) \text { in } \bar{Q}
$$

satisfies

$$
\left|\xi^{\sigma}(x, t)-\xi^{\sigma}(y, t)\right| \leq 2 M e^{-\theta t}|x-y| \quad \text { for }(x, t),(y, t) \in \bar{Q} .
$$

Proof. 1. To show (4.5), we set

$$
w(x)=v^{\lambda, \sigma}(x)+K_{\epsilon}|x|^{2} \quad \text { for } x \in \mathbb{R}^{n},
$$

where $v^{\lambda, \sigma}$ is the viscosity solution of (3.4) given by (3.7) and $K_{\epsilon}=K+\epsilon$ for $\epsilon \in(0, \theta / 2 \beta)$. Then $w$ is a viscosity solution of

$$
\begin{aligned}
& \lambda w(x)-\sigma \Delta w(x)+2 K_{\epsilon} n \sigma+\left(\alpha-4 \beta K_{\epsilon}\right) x \cdot D w(x)+\beta|D w(x)|^{2} \\
& -K_{\epsilon}\left(\lambda+2 \alpha-4 \beta K_{\epsilon}\right)|x|^{2}-f(x)=0 \quad \text { in } \mathbb{R}^{n} .
\end{aligned}
$$

We check that Proposition 3 of [1] is applicable. Note that $f(x)+B|x|^{2}$ is convex on $\mathbb{R}^{n}$. Since $K_{\epsilon}\left(2 \alpha-4 \beta K_{\epsilon}\right)>B$ for $\epsilon \in(0, \theta / 2 \beta)$ by $(4.2)$, the condition that

$$
\begin{aligned}
& (x, r, X) \mapsto \lambda r-\sigma \operatorname{tr} X^{-1}+2 K_{\epsilon} n \sigma+\left(\alpha-4 \beta K_{\epsilon}\right) x \cdot p+\beta|p|^{2} \\
& \quad-K_{\epsilon}\left(\lambda+2 \alpha-4 \beta K_{\epsilon}\right)|x|^{2}-f(x)
\end{aligned}
$$


is concave on $\mathbb{R}^{n} \times \mathbb{R} \times \mathcal{S}_{++}^{n}$ for each $p \in \mathbb{R}^{n}$ is satisfied, where $\mathcal{S}_{++}^{n}$ is the set of all $n \times n$ positive definite matrices. Since $K_{\epsilon}>0$ for $\epsilon \in(0, \theta / 2 \beta)$, the condition that

$$
\lim _{|x| \rightarrow \infty} \frac{w(x)}{|x|}=\infty
$$

is also satisfied. Hence, by Proposition 3 of [1], the convex envelope $w_{* *}$ of $w$ is a viscosity supersolution of (4.8). Hence, $w_{* *}(x)-K_{\epsilon}|x|^{2}$ is a viscosity supersolution of (3.4). By putting $g=v^{\lambda, \sigma}$ and $a=K_{\epsilon}$ in Lemma 4.4, we find a positive constant $C_{1}>0$ such that $-\left(w_{* *}(x)-K_{\epsilon}|x|^{2}\right) \leq C_{1}(1+|x|)$ for $x \in \mathbb{R}^{n}$. On the other hand, note that $v^{\lambda, \sigma}$ is a viscosity solution of (3.4) and there is a constant $C_{2}>0$ such that $v^{\lambda, \sigma}(x) \leq C_{2}(1+|x|)$ for $x \in \mathbb{R}^{n}$ by (3.8). Then, by Proposition 3.2, we have

$$
v^{\lambda, \sigma}(x) \leq w_{* *}(x)-K_{\epsilon}|x|^{2}, \quad x \in \mathbb{R}^{n} .
$$

Hence, $w \leq w_{* *}$ in $\mathbb{R}^{n}$. Since $w_{* *} \leq w$ in $\mathbb{R}^{n}$ by the definition of convex envelopes, we conclude that $w=w_{* *}$ in $\mathbb{R}^{n}$, so that $w$ is convex on $\mathbb{R}^{n}$. This implies that $v^{\lambda, \sigma}(x)-v^{\lambda, \sigma}(0)+K_{\epsilon}|x|^{2}$ is convex on $\mathbb{R}^{n}$. Letting $\epsilon, \lambda \searrow 0$, we see (4.5) by $(3.9)$.

2. Since $\xi^{\sigma} \in C^{2,1}(Q)$ by Remarks 2.3 and 3.3, we observe that $\xi^{\sigma}$ satisfies

$$
\xi_{t}^{\sigma}-\sigma \Delta \xi^{\sigma}+\alpha x \cdot D \xi^{\sigma}+\beta\left|D u^{\sigma}\right|^{2}-\beta\left|D v^{\sigma}\right|^{2}=0 \quad \text { in } Q .
$$

Since $0<\sigma<1$, we may assume that $D_{i} \xi^{\sigma} \in C^{2,1}(Q)$ for $i=1,2, \ldots, n$ by a suitable approximation by mollifiers. So, differentiating (4.9) with respect to $x$, we get

$$
\begin{aligned}
0= & \left(D \xi^{\sigma}\right)_{t}-\sigma \Delta\left(D \xi^{\sigma}\right)+\alpha D \xi^{\sigma}+D^{2} \xi^{\sigma}(\alpha x)+2 \beta D^{2} u^{\sigma} D u^{\sigma}-2 \beta D^{2} v^{\sigma} D v^{\sigma} \\
= & \left(D \xi^{\sigma}\right)_{t}-\sigma \Delta\left(D \xi^{\sigma}\right)+\alpha D \xi^{\sigma}+D^{2} \xi^{\sigma}(\alpha x)+2 \beta D^{2}\left(u^{\sigma}-v^{\sigma}\right) D u^{\sigma} \\
& +2 \beta D^{2} v^{\sigma} D\left(u^{\sigma}-v^{\sigma}\right) \\
= & \left(D \xi^{\sigma}\right)_{t}-\sigma \Delta\left(D \xi^{\sigma}\right)+\alpha D \xi^{\sigma}+D^{2} \xi^{\sigma}(\alpha x)+2 \beta D^{2} \xi^{\sigma} D u^{\sigma}+2 \beta D^{2} v^{\sigma} D \xi^{\sigma},
\end{aligned}
$$

so that we obtain in $Q$

$$
0=\left(D \xi^{\sigma}\right)_{t}-\sigma \Delta\left(D \xi^{\sigma}\right)+\alpha D \xi^{\sigma}+D^{2} \xi^{\sigma}\left(\alpha x+2 \beta D u^{\sigma}\right)+2 \beta D^{2} v^{\sigma} D \xi^{\sigma} .
$$

Note that

$$
2 D \xi^{\sigma} \cdot \Delta\left(D \xi^{\sigma}\right)=\Delta\left(\left|D \xi^{\sigma}\right|^{2}\right)-2 \sum_{i, j=1}^{n}\left|D_{i j} \xi^{\sigma}\right|^{2} \leq \Delta\left(\left|D \xi^{\sigma}\right|^{2}\right) .
$$

Hence, multiplying (4.10) by $2 D \xi^{\sigma}$, we get

$$
\begin{aligned}
0 \geq & \partial_{t}\left|D \xi^{\sigma}\right|^{2}-\sigma \Delta\left(\left|D \xi^{\sigma}\right|^{2}\right)+2 \alpha\left|D \xi^{\sigma}\right|^{2}+\left(\alpha x+2 \beta D u^{\sigma}\right) \cdot D\left|D \xi^{\sigma}\right|^{2} \\
& +4 \beta D^{2} v^{\sigma} D \xi^{\sigma} \cdot D \xi^{\sigma}
\end{aligned}
$$

Since $v^{\sigma} \in C^{2}\left(\mathbb{R}^{n}\right)$ satisfies (4.5), we have

$$
D^{2} v^{\sigma}(x) \geq-2 K I_{n} \quad \text { in } \mathbb{R}^{n},
$$


where $I_{n}$ is the $n \times n$ identity matrix on $\mathbb{R}^{n}$. Hence, we obtain in $Q$

$$
0 \geq \partial_{t}\left|D \xi^{\sigma}\right|^{2}-\sigma \Delta\left(\left|D \xi^{\sigma}\right|^{2}\right)+2(\alpha-4 \beta K)\left|D \xi^{\sigma}\right|^{2}+\left(\alpha x+2 \beta D u^{\sigma}\right) \cdot D\left|D \xi^{\sigma}\right|^{2} .
$$

Let $s>0$ be arbitrary. Define the function $z$ by

$$
z(x, t):=\left|D \xi^{\sigma}(x, t)\right|^{2} \quad \text { in } \mathbb{R}^{n} \times[s, \infty) .
$$

Since $\theta=\alpha-4 \beta K, z$ satisfies

$$
0 \geq z_{t}-\sigma \Delta z+\left(\alpha x+2 \beta D u^{\sigma}\right) \cdot D z+2 \theta z \quad \text { in } \mathbb{R}^{n} \times(s, \infty) .
$$

By (2.8) and (3.2), we see that

$$
z(x, t) \leq 2\left(\left|D u^{\sigma}(x, t)\right|^{2}+\left|D v^{\sigma}(x)\right|^{2}\right) \leq 4 M^{2} \text { in } \mathbb{R}^{n} \times[s, \infty) .
$$

Then, the function

$$
w(x, t):=4 M^{2} e^{-2 \theta(t-s)}-z(x, t) \text { in } \mathbb{R}^{n} \times[s, \infty)
$$

satisfies

$$
w_{t}-\sigma \Delta w+\left(\alpha x+2 \beta D u^{\sigma}\right) \cdot D w+2 \theta w \geq 0 \quad \text { in } \mathbb{R}^{n} \times(s, \infty)
$$

and

$$
0 \leq w(x, s) \text { in } \mathbb{R}^{n} .
$$

Hence, by the maximum principle of [8, Theorem 9 of Chap.2], we obtain

$$
0 \leq w(x, t) \text { for }(x, t) \in \mathbb{R}^{n} \times[s, \infty) .
$$

Letting $s \searrow 0$, we obtain $\left|D \xi^{\sigma}(x, t)\right| \leq 2 M e^{-\theta t}$ in $Q$, so that (4.7) holds for $(x, t),(y, t) \in Q$. It follows from (2.8) and (3.2) that inequality (4.7) holds for $x, y \in \mathbb{R}^{n}$ and $t=0$. Hence, we obtain $(4.7)$ for $(x, t),(y, t) \in \bar{Q}$. The proof is complete.

Proof of Theorem 4.1. 1. By Propositions 2.5 and 3.4, we can find a sequence $\left\{\sigma_{j}\right\}$, a constant $c \in \mathbb{R}$ and a viscosity solution $v \in \operatorname{Lip}\left(\mathbb{R}^{n}\right)$ of (1.6) such that

$$
\begin{aligned}
& c^{\sigma_{j}} \rightarrow c, \quad v^{\sigma_{j}} \rightarrow v \text { locally uniformly on } \mathbb{R}^{n}, \\
& u^{\sigma_{j}} \rightarrow u \text { locally uniformly on } \bar{Q} \text {. }
\end{aligned}
$$

By (4.5) and (4.12), we have

$$
v(x)+K|x|^{2} \text { is convex on } \mathbb{R}^{n},
$$

so that (i) holds.

2. We show that the function

$$
\xi(x, t):=u(x, t)-(c t+v(x)) \text { in } \bar{Q}
$$

is differentiable with respect to $(x, t)$ almost everywhere in $Q$, and satisfies

$$
\left|\xi_{t}(x, t)\right| \leq 2 M(\alpha|x|+2 \beta M) e^{-\theta t} \text { for a.e. }(x, t) \in Q .
$$

Indeed, by Proposition 2.2, the function $u$ is differentiable with respect to $x$ and $t$ almost everywhere in $Q$. Hence, $u$ satisfies (1.4) almost everywhere in $Q$. Similarly, since $v \in \operatorname{Lip}\left(\mathbb{R}^{n}\right)$, the pair $(c, v)$ satisfies (1.6) almost everywhere 
in $\mathbb{R}^{n}$. Therefore, $\xi$ of $(4.15)$ is differentiable with respect to $x$ and $t$ almost everywhere in $Q$, and satisfies

$$
\xi_{t}+\alpha x \cdot D \xi+\beta|D u|^{2}-\beta|D v|^{2}=0 \text { for a.e. }(x, t) \in Q .
$$

On the other hand, by (4.7), (4.12) and (4.13), we have

$$
|\xi(x, t)-\xi(y, t)| \leq 2 M e^{-\theta t}|x-y| \quad \text { for }(x, t),(y, t) \in \bar{Q},
$$

so that

$$
|D \xi(x, t)| \leq 2 M e^{-\theta t} \text { for a.e. }(x, t) \in Q .
$$

Then, it follows from (2.8) and (3.2) that

$$
\begin{aligned}
& \left.|\alpha x \cdot D \xi(x, t)+\beta| D u(x, t)\right|^{2}-\beta|D v(x)|^{2} \mid \\
& \quad=|\alpha x \cdot D \xi(x, t)+\beta(D u(x, t)+D v(x)) \cdot D \xi(x, t)| \\
& \quad \leq 2 M(\alpha|x|+2 \beta M) e^{-\theta t} \text { for a.e. }(x, t) \in Q .
\end{aligned}
$$

Hence, by (4.17), we obtain (4.16).

3. Let $s, t>0$ be given constants. We integrate (4.16) over $B(x, \epsilon) \times[t, t+s]$, where $x \in \mathbb{R}^{n}$ and $\epsilon>0$. Then, by (4.16), we get

$$
\begin{aligned}
& \int_{B(x, \epsilon)}|\xi(y, t+s)-\xi(y, t)| d y \leq \int_{B(x, \epsilon) \times[t, t+s]}\left|\xi_{\tau}(y, \tau)\right| d \tau d y \\
& \quad \leq \frac{2 M}{\theta}\left(e^{-\theta t}-e^{-\theta(t+s)}\right) \int_{B(x, \epsilon)}(\alpha|y|+2 \beta M) d y .
\end{aligned}
$$

Hence, we obtain

$$
\begin{aligned}
& \frac{1}{\epsilon^{n}} \int_{B(x, \epsilon)}|\xi(y, t+s)-\xi(y, t)| d y \\
& \quad \leq \frac{1}{\epsilon^{n}} \frac{2 M}{\theta}\left(e^{-\theta t}-e^{-\theta(t+s)}\right) \int_{B(x, \epsilon)}(\alpha|y|+2 \beta M) d y
\end{aligned}
$$

Letting $\epsilon \searrow 0$, we see that

$$
|\xi(x, t+s)-\xi(x, t)| \leq \frac{2 M}{\theta}\left(e^{-\theta t}-e^{-\theta(t+s)}\right)(\alpha|x|+2 \beta M)
$$

for all $s, t \geq 0, x \in \mathbb{R}^{n}$. Hence the function

$$
\zeta(x):=\lim _{t \rightarrow \infty} \xi(x, t)
$$

exists for all $x \in \mathbb{R}^{n}$. By (4.18), we have $\zeta \equiv a$ for some constant $a \in \mathbb{R}$. Letting $s \rightarrow \infty$ in $(4.19)$, we get

$$
|a-(u(x, t)-c t-v(x))| \leq \frac{2 M}{\theta} e^{-\theta t}(\alpha|x|+2 \beta M) \quad \text { in } \mathbb{R}^{n} \times[0, \infty) .
$$

Note that $(c, v(\cdot)+a)$ is also a viscosity solution of (1.6). Hence rewriting $v(\cdot)+a$ by $v(\cdot)$, we have shown the result. 
Next, we give an example to illustrate that we cannot improve the rate of convergence $e^{-\theta t}$ in (1.8) in general.

Theorem 4.6. Let $\alpha, \beta>0$ in (1.4). Then, for any constant $I_{0} \in\left[0, \alpha^{2} / 4 \beta\right)$, there are $f, u_{0} \in \operatorname{Lip}\left(\mathbb{R}^{n}\right)$ satisfying the following:

$$
\begin{aligned}
& I(f)=I_{0} . \\
& \quad-\delta|x| e^{-\theta t}+\frac{\beta \delta^{2}}{2 \theta} e^{-2 \theta t} \leq u(x, t)-v(x) \\
& \quad \leq-\delta \min \{|x|, 1\} e^{-\theta t}+\frac{\beta \delta^{2}}{2 \theta} e^{-2 \theta t} \text { in } \mathbb{R}^{n} \times[0, \infty),
\end{aligned}
$$

where $\theta=\sqrt{\alpha^{2}-4 \beta I_{0}}$ and $\delta \in(0, \theta / 2 \beta)$ is a constant; $v \in \operatorname{Lip}\left(\mathbb{R}^{n}\right)$ is a viscosity solution of (1.6) for $c=0$. Hence, (1.9) holds.

In the following, for $\rho \in C\left(\mathbb{R}^{n}\right)$ and $x \in \mathbb{R}^{n}$, we denote $D^{+} \rho(x)$ and $D^{-} \rho(x)$ by the super-differential set and the sub-differential set of $\rho$ at $x$, respectively. For $\chi \in C\left(\mathbb{R}^{n} \times(0, \infty)\right)$ and $(x, t) \in \mathbb{R}^{n} \times(0, \infty)$, we define $D_{x}^{ \pm} \chi(x, t)$ and $D_{t}^{ \pm} \chi(x, t)$ similarly. Here, the subscripts $x$ and $t$ denotes the "differentiation" with respect to $x$ and $t$, respectively.

Proof of Theorem 4.6. 1. We define $f$ by

$$
f(x)= \begin{cases}-I_{0}|x|^{2} & \text { for }|x| \leq 1, \\ -2 \alpha K_{0}|x|+4 \beta K_{0}^{2} & \text { for } 1<|x|,\end{cases}
$$

where $K_{0}=(\alpha-\theta) / 4 \beta$. Since $4 \beta K_{0}^{2}-2 \alpha K_{0}+I_{0}=0$, we see that $f \in \operatorname{Lip}\left(\mathbb{R}^{n}\right)$.

Next, it is easy to see that $f(x)+\mu|x|^{2}$ is not convex on $\mathbb{R}^{n}$ for $\mu<I_{0}$. Note that

$$
f(x)+I_{0}|x|^{2}= \begin{cases}0 & \text { for }|x| \leq 1, \\ 4 \beta K_{0}(|x|-1)\left(K_{0}^{\prime}|x|-K_{0}\right) & \text { for } 1<|x|,\end{cases}
$$

where $K_{0}^{\prime}=(\alpha+\theta) / 4 \beta$. Hence, $f(x)+I_{0}|x|^{2}$ is convex on $\mathbb{R}^{n}$, so that $f$ satisfies (4.20). Let

$$
v(x)= \begin{cases}-K_{0}|x|^{2} & \text { for }|x| \leq 1 \\ -2 K_{0}|x|+K_{0} & \text { for } 1<|x|\end{cases}
$$

Then, $v \in C^{1}\left(\mathbb{R}^{n}\right)$ and $v$ is a classical solution of (1.6) for $c=0$.

2. We define $\eta \in \operatorname{Lip}\left(\mathbb{R}^{n} \times[0, \infty)\right)$ by

$$
\eta(x, t)=v(x)-\delta|x| e^{-\theta t}+\frac{\beta \delta^{2}}{2 \theta} e^{-2 \theta t} \quad \text { in } \mathbb{R}^{n} \times[0, \infty) .
$$

Choose $u_{0} \in \operatorname{Lip}\left(\mathbb{R}^{n}\right)$ so that

$$
v(x)-\delta|x|+\frac{\beta \delta^{2}}{2 \theta} \leq u_{0}(x) \leq v(x)-\delta \min \{|x|, 1\}+\frac{\beta \delta^{2}}{2 \theta} \quad \text { in } \mathbb{R}^{n} .
$$

Since $D_{x}^{+} \eta(0, t)=\left\{-\delta e^{-\theta t} z \mid z \in B(0,1)\right\}$, we see easily that $\eta$ is a viscosity subsolution of (1.4). Since $\eta(x, 0) \leq u_{0}(x)$ for $x \in \mathbb{R}^{n}$, we conclude that $\eta \leq u$ in $\mathbb{R}^{n} \times[0, \infty)$ by Proposition 2.1 . 
3. We define $\rho \in \operatorname{Lip}\left(\mathbb{R}^{n} \times[0, \infty)\right)$ by

$$
\rho(x, t)=v(x)-\delta \min \{|x|, 1\} e^{-\theta t}+\frac{\beta \delta^{2}}{2 \theta} e^{-2 \theta t} \quad \text { in } \mathbb{R}^{n} \times[0, \infty) .
$$

We will show that, for $(x, t) \in \mathbb{R}^{n} \times(0, \infty), p \in D_{x}^{-} \rho(x, t)$ and $r \in D_{t}^{-} \rho(x, t)$

$$
r+\alpha x \cdot p+\beta|p|^{2} \geq f(x) .
$$

Since $D_{x}^{-} \rho(0, t)=\emptyset$, we have nothing to prove in (4.22) for $|x|=0$. For $0<|x|<1$, we can show (4.22) easily. Since $D_{x}^{-} \rho(x, t)=\left\{-2 K x-\delta m e^{-\theta t} x \mid 0 \leq m \leq 1\right\}$ for $|x|=1$, we have for $(x, t) \in \mathbb{R}^{n} \times(0, \infty), p \in D_{x}^{-} \rho(x, t)$ and $r \in D_{t}^{-} \rho(x, t)$

$$
\begin{aligned}
& r+\alpha x \cdot p+\beta|p|^{2}=f(x)+(1-m) \delta e^{-2 \theta t}\left[\theta e^{\theta t}-\beta \delta(1+m)\right] \\
& \quad \geq f(x)+(1-m) \delta e^{-2 \theta t}(\theta-2 \beta \delta) \geq f(x) .
\end{aligned}
$$

Hence, (4.22) holds in this case. When $|x|>1$, we can show (4.22) similarly. Thus, $\rho$ is a viscosity supersolution of (1.4). Since $u_{0} \leq \rho(\cdot, 0)$ in $\mathbb{R}^{n}$, we conclude that $u \leq \rho$ in $\mathbb{R}^{n} \times[0, \infty)$ by Proposition 2.1 . The proof is complete.

Finally, we give an example which shows that when $I(f)=\alpha^{2} / 4 \beta$, the rate of convergence in (1.3) changes according to initial data $u_{0} \in \operatorname{Lip}\left(\mathbb{R}^{n}\right)$. This is very contrary to the case $I(f)<\alpha^{2} / 4 \beta$. Let

$$
w(x)= \begin{cases}|x|^{2} & \text { for }|x| \leq 1, \\ 2|x|-1 & \text { for } 1<|x|,\end{cases}
$$

Note that $w \in C^{1}\left(\mathbb{R}^{n}\right) \cap \operatorname{Lip}\left(\mathbb{R}^{n}\right)$. The following theorem is a special case of [11].

Theorem 4.7. Let $\alpha, \beta>0$ in (1.4). Then, the function

$$
f(x)=-\frac{\alpha^{2}}{4 \beta} w(x) \quad \text { in } \mathbb{R}^{n}
$$

satisfies the following:

(i) $I(f)=\alpha^{2} / 4 \beta$.

(ii) If $u_{0} \equiv 0$, then

$$
\begin{aligned}
\frac{\alpha}{4 \beta(\alpha t+1)} \min \left\{|x|^{2}, 1\right\} \leq u(x, t)-v(x) \leq & \frac{\alpha}{4 \beta(\alpha t+1)} w(x) \\
& \text { in } \mathbb{R}^{n} \times[0, \infty),
\end{aligned}
$$

where $v(x)=-(\alpha / 4 \beta) w(x)$ in $\mathbb{R}^{n}$. The function $v \in \operatorname{Lip}\left(\mathbb{R}^{n}\right)$ is a viscosity solution of (1.6) for $c=0$.

(iii) If $u_{0}=v$ in $\mathbb{R}^{n}$, then

$$
u(x, t)-(c t+v(x))=0 \quad \text { in } \mathbb{R}^{n} \times[0, \infty),
$$

where $v$ is the function of (ii).

Remark 4.8. In Theorem 4.7, we have $\mathcal{A}=B(0,1)$, where $\mathcal{A}$ is the Aubry set of Remark 4.3. This is contrary to the case $I(f)<\alpha^{2} / 4 \beta$ in Remark 4.3. 
Proof of Theorem 4.7. 1. We see that $f \in \operatorname{Lip}\left(\mathbb{R}^{n}\right)$ and $I(f)=\alpha^{2} / 4 \beta$. Then, $v \in C^{1}\left(\mathbb{R}^{n}\right)$ and $v$ is a classical solution of (1.6) for $c=0$. Hence, we obtain (i).

2. We define $\eta \in C\left(\mathbb{R}^{n} \times[0, \infty)\right)$ by

$$
\eta(x, t):=v(x)+\frac{\alpha}{4 \beta(\alpha t+1)} z(x) \text { in } \mathbb{R}^{n} \times[0, \infty),
$$

where $z(x)=\min \left\{|x|^{2}, 1\right\}$ in $\mathbb{R}^{n}$. Since $D^{+} z(x)=\{k x \mid 0 \leq k \leq 2\}$, we see easily that $\eta$ is a viscosity subsolution of (1.4). Since $\eta(\cdot, 0) \leq 0=u_{0}$ in $\mathbb{R}^{n}$, we conclude that $\eta \leq u$ in $\mathbb{R}^{n} \times[0, \infty)$ by Proposition 2.1 .

Next, we define $\rho \in C\left(\mathbb{R}^{n} \times[0, \infty)\right)$ by

$$
\rho(x, t):=v(x)+\frac{\alpha}{4 \beta(\alpha t+1)} w(x) \text { in } \mathbb{R}^{n} \times[0, \infty) .
$$

We see easily that $\rho$ is a viscosity supersolution of (1.4). Since $u_{0}=0=\rho(\cdot, 0)$ in $\mathbb{R}^{n}$, we conclude that $u \leq \rho$ in $\mathbb{R}^{n} \times[0, \infty)$ by Proposition 2.1 . Hence, we obtain (ii).

3. By uniqueness of viscosity solutions of (1.4) and (1.5) in Proposition 2.1, we have (iii) easily.

\section{Proofs of results of Section 2}

For $T>0$, we write

$$
Q_{T}:=\mathbb{R}^{n} \times(0, T), \quad R_{T}:=\mathbb{R}^{n} \times[0, T) .
$$

Proof of Proposition 2.1. 1. Fix any $T>0$ and we show that

$$
u(x, t)-v(y, t) \leq M|x-y| \text { for }(x, t),(y, t) \in \bar{Q}_{T},
$$

which is enough to conclude Proposition 2.1. By replacing $u$ and $v$ by their upper and lower semi-continuous envelopes, respectively, we may assume that $u,-v \in$ $\operatorname{USC}\left(R_{T}\right)$. Then $(2.7)$ reads that

$$
u(x, 0) \leq u_{0}(x) \leq v(x, 0) \quad \text { in } \mathbb{R}^{n} .
$$

To show (5.1) we argue by contradiction, and hence assume that

$$
\sup \left\{u(x, t)-v(y, t)-M|x-y| \mid(x, t),(y, t) \in R_{T}\right\}>0,
$$

and will get a contradiction.

2. Let $\kappa>1$ and $\gamma, \delta, \epsilon \in(0,1)$. We define the functions $\Phi \in \operatorname{USC}\left(R_{T} \times R_{T}\right)$ and $\Psi \in \operatorname{USC}\left(\mathbb{R}^{n} \times[0, T) \times \mathbb{R}^{n}\right)$, respectively, by

$$
\begin{aligned}
\Phi(x, t, y, s)= & u(x, t)-v(y, s)-M\langle x-y\rangle_{\gamma}-\kappa|t-s|^{2}-\delta\left(|x|^{2}+|y|^{2}\right) \\
& -\frac{\epsilon}{T-t}-\frac{\epsilon}{T-s}, \\
\Psi(x, t, y)= & u(x, t)-v(y, t)-M\langle x-y\rangle_{\gamma}-\delta\left(|x|^{2}+|y|^{2}\right)-\frac{2 \epsilon}{T-t} .
\end{aligned}
$$

Here and henceforth $\langle x\rangle_{\gamma}$ denotes $\left(|x|^{2}+\gamma^{2}\right)^{\frac{1}{2}}$ for $x \in \mathbb{R}^{n}$ and $\gamma>0$. Taking into account of the dependence on the parameters $\gamma, \delta, \epsilon, \kappa$, we write as well 
$\Phi(x, t, y, s ; \gamma, \delta, \epsilon, \kappa)$ and $\Psi(x, t, y ; \gamma, \delta, \epsilon)$ for $\Phi(x, t, y, s)$ and $\Psi(x, t, y)$, respectively. It is obvious that the functions $\Phi(\cdot ; \gamma, \delta, \epsilon, \kappa)$ and $\Psi(\cdot ; \gamma, \delta, \epsilon)$ attain their maxima.

We denote by $\mathcal{M}(\gamma, \delta, \epsilon, \kappa)$ the set of the maximum points of the function $\Phi(\cdot ; \gamma, \delta, \epsilon, \kappa)$ and, similarly, by $\mathcal{M}(\gamma, \delta, \epsilon)$ the set of the maximum points of the function $\Psi(\cdot ; \gamma, \delta, \epsilon)$.

In view of $(5.2)$, we may choose a constant $\theta \in(0,1)$ so that $\max \Psi(\cdot ; \theta, \theta, \theta)>$ 0 . Observe that, since

$$
\Psi(x, t, y ; \gamma, \delta, \epsilon)=\Phi(x, t, y, t ; \gamma, \delta, \epsilon, \kappa) \quad \text { for } x, y \in \mathbb{R}^{n}, t \in[0, T),
$$

we have

$$
\max \Phi(\cdot ; \gamma, \delta, \epsilon, \kappa) \geq \max \Psi(\cdot ; \gamma, \delta, \epsilon) .
$$

Hence, for $\gamma, \delta, \epsilon \in(0, \theta]$ and $\kappa>1$, we have

$$
\max \Phi(\cdot ; \gamma, \delta, \epsilon, \kappa) \geq \max \Psi(\cdot ; \gamma, \delta, \epsilon) \geq \max \Psi(\cdot ; \theta, \theta, \theta)>0 .
$$

3. In what follows we are always concerned with those $\gamma, \delta, \epsilon$ which satisfy

$$
\gamma, \delta, \epsilon \in(0, \theta] \text {. }
$$

Let $(x, t, y, s) \in \mathcal{M}(\gamma, \delta, \epsilon, \kappa)$. Using (2.6) and (5.3), we get

$$
\begin{aligned}
0< & C\left(1+\left(|x|^{2}+|y|^{2}\right)^{1 / 2}\right)-M|x-y|-\delta\left(|x|^{2}+|y|^{2}\right)-\kappa|t-s|^{2} \\
& -\frac{\epsilon}{T-t}-\frac{\epsilon}{T-s} \\
\leq & \frac{\delta}{2}\left(|x|^{2}+|y|^{2}\right)+C+\frac{C^{2}}{2 \delta}-M|x-y|-\delta\left(|x|^{2}+|y|^{2}\right)-\kappa|t-s|^{2} \\
& -\frac{\epsilon}{T-t}-\frac{\epsilon}{T-s}
\end{aligned}
$$

for some constant $C>0$. Consequently, we have

$$
\begin{gathered}
\frac{\delta}{2}\left(|x|^{2}+|y|^{2}\right)+M|x-y|+\frac{\epsilon}{T-t}+\frac{\epsilon}{T-s}+\kappa|t-s|^{2} \leq C_{\delta} \\
:=C\left(1+\frac{C}{2 \delta}\right)
\end{gathered}
$$

for all $(x, t, y, s) \in \mathcal{M}(\gamma, \delta, \epsilon, \kappa)$.

4. Observe that there is a $\kappa_{0} \equiv \kappa_{0}(\gamma, \delta, \epsilon)>1$ such that for any $(x, t, y, s) \in$ $\mathcal{M}(\gamma, \delta, \epsilon, \kappa)$

$$
t>0, s>0 \quad \text { if } \kappa>\kappa_{0} .
$$

To see this, we argue by contradiction. Suppose that there are sequences $\kappa_{j} \rightarrow \infty$ and $\left(t_{j}, x_{j}, s_{j}, y_{j}\right) \in \mathcal{M}\left(\gamma, \delta, \epsilon, \kappa_{j}\right)$, with $j \in \mathbb{N}$, such that

$$
t_{j}=0 \text { or } s_{j}=0 \quad \text { for all } j \in \mathbb{N} \text {. }
$$


Then, by (5.4), we see that $t_{j}, s_{j} \rightarrow 0$ as $j \rightarrow \infty$. Noting that the set $\cup_{j \in \mathbb{N}} \mathcal{M}$ $\left(\gamma, \delta, \epsilon, \kappa_{j}\right)$ is bounded by (5.4), we may assume that $\left(x_{j}, y_{j}\right) \rightarrow(\bar{x}, \bar{y})$ as $j \rightarrow \infty$ for some $(\bar{x}, \bar{y}) \in \mathbb{R}^{n} \times \mathbb{R}^{n}$. Since

$$
\begin{aligned}
& \max \Psi(\cdot ; \gamma, \delta, \epsilon) \leq \Phi\left(x_{j}, t_{j}, y_{j}, s_{j} ; \gamma, \delta, \epsilon, \kappa_{j}\right) \\
& \quad \leq u\left(x_{j}, t_{j}\right)-v\left(y_{j}, s_{j}\right)-M\left\langle x_{j}-y_{j}\right\rangle_{\gamma}-\delta\left(\left|x_{j}\right|^{2}+\left|y_{j}\right|^{2}\right)-\frac{\epsilon}{T-t_{j}}-\frac{\epsilon}{T-s_{j}} \\
& \quad \rightarrow \Psi(\bar{x}, 0, \bar{y} ; \gamma, \delta, \epsilon)
\end{aligned}
$$

as $j \rightarrow \infty$, we find that $(\bar{x}, 0, \bar{y}) \in \mathcal{M}(\gamma, \delta, \epsilon)$, and hence $\Psi(\bar{x}, 0, \bar{y} ; \gamma, \delta, \epsilon)>0$. Therefore, we have

$0<\Psi(\bar{x}, 0, \bar{y} ; \gamma, \delta, \epsilon)<u_{0}(\bar{x})-u_{0}(\bar{y})-M\langle\bar{x}-\bar{y}\rangle_{\gamma} \leq L_{u_{0}}|\bar{x}-\bar{y}|-L_{u_{0}}\langle\bar{x}-\bar{y}\rangle_{\gamma}<0$.

This is a contradiction, which shows that there is a $\kappa_{0}>1$, depending on $\gamma, \delta, \epsilon$, for which (5.5) holds.

5. Fix $\kappa>\kappa_{0}$ and $(\hat{x}, \hat{t}, \hat{y}, \hat{s}) \in \mathcal{M}(\gamma, \delta, \epsilon, \kappa)$, so that $\hat{t}>0$ and $\hat{s}>0$. Then there is a matrix $\hat{X} \in \mathcal{S}^{n}$ for which we have

$$
\begin{aligned}
& \frac{\epsilon}{(T-\hat{t})^{2}}+2 \kappa(\hat{t}-\hat{s})-\sigma(\operatorname{tr} \hat{X}+2 \delta n)+\alpha \hat{x} \cdot(M \hat{p}+2 \delta \hat{x})+H(M \hat{p}+2 \delta \hat{x}) \leq f(\hat{x}) \\
& -\frac{\epsilon}{(T-\hat{s})^{2}}+2 \kappa(\hat{t}-\hat{s})-\sigma(\operatorname{tr} \hat{X}-2 \delta n)+\alpha \hat{y} \cdot(M \hat{p}-2 \delta \hat{y})+H(M \hat{p}-2 \delta \hat{y}) \geq f(\hat{y}),
\end{aligned}
$$

where $\hat{p}:=(\hat{x}-\hat{y}) /\langle\hat{x}-\hat{y}\rangle_{\gamma}$. From these we get

$$
\begin{aligned}
& \frac{2 \epsilon}{T^{2}}-4 n \delta \sigma+2 \alpha \delta\left(|\hat{x}|^{2}+|\hat{y}|^{2}\right)+\alpha M \frac{|\hat{x}-\hat{y}|^{2}}{\langle\hat{x}-\hat{y}\rangle_{\gamma}}+H(M \hat{p}+2 \delta \hat{x}) \\
& \quad-H(M \hat{p}-2 \delta \hat{y}) \leq f(\hat{x})-f(\hat{y}) \leq L_{f}|\hat{x}-\hat{y}| .
\end{aligned}
$$

Now, by (5.4), we have

$$
\frac{\delta}{2}\left(|\hat{x}|^{2}+|\hat{y}|^{2}\right)+\kappa|\hat{t}-\hat{s}|^{2}+\frac{\epsilon}{T-\hat{t}}+\frac{\epsilon}{T-\hat{s}} \leq C\left(1+\frac{C}{2 \delta}\right)
$$

and find that there is a limit point $(\bar{t}, \bar{t}, \bar{x}, \bar{y}, \bar{p}) \in[0, T) \times[0, T) \times \mathbb{R}^{n} \times \mathbb{R}^{n} \times \mathbb{R}^{n}$ of $(\hat{s}, \hat{t}, \hat{x}, \hat{y}, \hat{p})$, which depends on $\delta$, along a sequence of $(\gamma, \kappa)$ such that $\gamma \searrow 0$ and $\kappa \rightarrow \infty$. Then, we have

$$
\lim _{\delta \downarrow 0} \frac{|\hat{x}-\hat{y}|^{2}}{\langle\hat{x}-\hat{y}\rangle_{\gamma}} \geq|\bar{x}-\bar{y}| .
$$

From (5.6), we get

$$
\begin{aligned}
& \frac{2 \epsilon}{T^{2}}+2 \alpha \delta\left(|\bar{x}|^{2}+|\bar{y}|^{2}\right)+\alpha M|\bar{x}-\bar{y}| \leq 4 n \delta \sigma+H(M \bar{p}-2 \delta \bar{y})-H(M \bar{p}+2 \delta \bar{x}) \\
& \quad+L_{f}|\bar{x}-\bar{y}| .
\end{aligned}
$$

Since $\alpha M \geq L_{f}$, we get

$$
\frac{2 \epsilon}{T^{2}}+2 \alpha \delta\left(|\bar{x}|^{2}+|\bar{y}|^{2}\right) \leq 4 n \delta \sigma+H(M \bar{p}-2 \delta \bar{y})-H(M \bar{p}+2 \delta \bar{x}) .
$$


By (5.4) and the definition of $\bar{p}$, note that

$$
|\bar{p}| \leq 1, \quad \delta(|\bar{x}|+|\bar{y}|) \leq C_{1}
$$

for some constant $C_{1}>0$. Then, the right-hand side of (5.7) is bounded by a constant. This implies that

$$
\delta\left(|\bar{x}|^{2}+|\bar{y}|^{2}\right) \leq C_{2}
$$

for some constant $C_{2}>0$. Hence, we observe that as $\delta \searrow 0$ along a sequence,

$$
\bar{t} \rightarrow \tilde{t}, \quad \bar{p} \rightarrow \tilde{p}, \quad \delta(|\bar{x}|+|\bar{y}|) \leq \sqrt{\delta} \sqrt{2 \delta\left(|\bar{x}|^{2}+|\bar{y}|^{2}\right)} \rightarrow 0
$$

for some $\tilde{p} \in \mathbb{R}^{n}$. From (5.7), we get

$$
\frac{2 \epsilon}{T^{2}} \leq H(M \tilde{p})-H(M \tilde{p})=0 .
$$

This is a contradiction, which was to be shown.

Proof of Proposition 2.2. 1. Uniqueness follows from Proposition 2.1. To show existence, we use the Perron method. Let $y \in \mathbb{R}^{n}, \epsilon>0$, and $A>0$, and set

$$
g(x, t)=u_{0}(y)+M\langle x-y\rangle_{\epsilon}+A t \quad \text { for }(x, t) \in \bar{Q},
$$

where $M$ is the constant of (2.5). Compute that for $(x, t) \in Q$,

$$
\Delta_{x}\langle x-y\rangle_{\epsilon} \leq \frac{n}{\epsilon}, \quad x \cdot D_{x}\langle x-y\rangle_{\epsilon}=\frac{x \cdot(x-y)}{\langle x-y\rangle_{\epsilon}} \geq\langle x-y\rangle_{\epsilon}-\epsilon-|y| .
$$

Let $C_{M}=\max \{|H(p)||| p \mid \leq M\}$. Since $\alpha M \geq L_{f}$, we have

$$
\begin{aligned}
& {\left[g_{t}-\sigma \Delta g+\alpha x \cdot D g+H(D g)-f\right](x, t)} \\
& \quad \geq A-\frac{M n \sigma}{\epsilon}+M \alpha\left[\langle x-y\rangle_{\epsilon}-\epsilon-|y|\right]-C_{M}-f(x) \\
& \quad \geq A-\frac{M n \sigma}{\epsilon}+M \alpha\left[\langle x-y\rangle_{\epsilon}-\epsilon-|y|\right]-C_{M}-|f(y)|-L_{f}|x-y| \\
& \quad \geq A-\frac{M n \sigma}{\epsilon}-M \alpha(\epsilon+|y|)-C_{M}-|f(y)| .
\end{aligned}
$$

For each $y \in \mathbb{R}^{n}$ and $\epsilon \in(0,1)$ we set

$$
A^{\sigma}(y, \epsilon)=\frac{M n \sigma}{\epsilon}+M \alpha(\epsilon+|y|)+C_{M}+|f(y)|,
$$

and define the functions $\theta^{\sigma} \in C^{\infty}(\bar{Q})$, parameterized by $y, \epsilon$, by

$$
\theta^{\sigma}(x, t ; y, \epsilon)=u_{0}(y)+M\langle x-y\rangle_{\epsilon}+A^{\sigma}(y, \epsilon) t .
$$

Note that, for any $y \in \mathbb{R}^{n}$ and $\epsilon \in(0,1)$, the function $\theta^{\sigma}(\cdot, y, \epsilon)$ are supersolutions of (2.1),

$\theta^{\sigma}(x, t ; y, \epsilon) \geq u_{0}(x)-L_{u_{0}}|x-y|+M\langle x-y\rangle_{\epsilon}+A^{\sigma}(y, \epsilon) t \geq u_{0}(x)$ for $(x, t) \in \bar{Q}$, and

$$
\theta^{\sigma}(x, 0 ; x, \epsilon)=u_{0}(x)+M \epsilon \quad \text { for } x \in \mathbb{R}^{n}
$$


2. We define $U^{\sigma} \in \operatorname{USC}(\bar{Q})$ by

$$
U^{\sigma}(x, t)=\inf \left\{\theta^{\sigma}(x, t ; y, \epsilon) \mid y \in \mathbb{R}^{n}, \epsilon \in(0,1)\right\} .
$$

Then the lower semicontinuous envelope $\left(U^{\sigma}\right)_{*}$ of $U^{\sigma}$ is a viscosity supersolution of (2.1) and

$$
u_{0}(x) \leq\left(U^{\sigma}\right)_{*}(x, t) \leq U^{\sigma}(x, t) \quad \text { for all }(x, t) \in \bar{Q} .
$$

Also, noting that

$$
u_{0}(x) \leq U^{\sigma}(x, 0) \leq \theta(x, 0 ; x, \epsilon, \sigma)=u_{0}(x)+M \epsilon \quad \text { for } x \in \mathbb{R}^{n}, \epsilon \in(0,1),
$$

we see that

$$
U^{\sigma}(x, 0)=\left(U^{\sigma}\right)_{*}(x, 0)=u_{0}(x) \quad \text { for } x \in \mathbb{R}^{n} .
$$

3. Similarly we define the functions $\chi^{\sigma} \in C^{\infty}(\bar{Q})$, parameterized by $y \in$ $\mathbb{R}^{n}$ and $\epsilon \in(0,1)$, by

$$
\chi^{\sigma}(x, t ; y, \epsilon)=u_{0}(y)-M\langle x-y\rangle_{\epsilon}-A^{\sigma}(y, \epsilon) t,
$$

and the function $V^{\sigma} \in \operatorname{LSC}(\bar{Q})$ by

$$
V^{\sigma}(x, t)=\sup \left\{\chi^{\sigma}(x, t ; y, \epsilon) \mid y \in \mathbb{R}^{n}, \epsilon \in(0,1)\right\} .
$$

Then we see as before that $\left(V^{\sigma}\right)^{*}$ is a viscosity subsolution of (2.1) and that for $(x, t) \in \bar{Q}$,

$$
u_{0}(x) \geq\left(V^{\sigma}\right)^{*}(x, t) \geq V^{\sigma}(x, t), \quad V^{\sigma}(x, 0)=\left(V^{\sigma}\right)^{*}(x, 0)=u_{0}(x) .
$$

4. Define $u^{\sigma}: \bar{Q} \rightarrow \mathbb{R}^{n}$ by

$$
\begin{array}{r}
u^{\sigma}(x, t)=\sup \left\{w(x, t) \mid V^{\sigma} \leq w \leq U^{\sigma} \text { on } \bar{Q}, \text { and } w\right. \\
\text { is a viscosity subsolution of }(2.1)\} .
\end{array}
$$

Then $\left(u^{\sigma}\right)^{*}$ and $\left(u^{\sigma}\right)_{*}$ are a viscosity subsolution and a viscosity supersolution of (2.1), respectively, and

$$
V^{\sigma} \leq u^{\sigma} \leq U^{\sigma} \quad \text { on } \bar{Q}
$$

In particular, we see that as $t \rightarrow 0$,

$u^{\sigma}(x, t),\left(u^{\sigma}\right)^{*}(x, t),\left(u^{\sigma}\right)_{*}(x, t) \rightarrow u_{0}(x) \quad$ locally uniformly on $\mathbb{R}^{n}$.

5. Next we show that $u^{\sigma}(x, t)$, as functions of $x$, grows at most linearly as $|x| \rightarrow \infty$. Indeed, for all $(x, t) \in \bar{Q}$ and $\epsilon \in(0,1)$, we have

$$
\begin{aligned}
& \left|u^{\sigma}(x, t)-u_{0}(x)\right| \leq \max \left\{U^{\sigma}(x, t)-u_{0}(x), u_{0}(x)-V^{\sigma}(x, t)\right\} \\
& \quad \leq \max \left\{\theta^{\sigma}(x, t ; x, \epsilon)-u_{0}(x), u_{0}(x)-\chi^{\sigma}(x, t ; x, \epsilon)\right\} \\
& \quad \leq M \epsilon+A^{\sigma}(x, \epsilon) t=M \epsilon+t\left[\frac{M n \sigma}{\epsilon}+M \alpha(\epsilon+|x|)+C_{M}+|f(x)|\right] .
\end{aligned}
$$


Now, we apply Proposition 2.1, with $\left(u^{\sigma}\right)^{*}$ and $\left(u^{\sigma}\right)_{*}$ in place of $u$ and $v$, respectively, to find that $\left(u^{\sigma}\right)^{*} \leq\left(u^{\sigma}\right)_{*}$ in $\bar{Q}$, that is, $u^{\sigma} \in C(\bar{Q})$. Next we apply Proposition 2.1, to deduce (2.8). Finally, by (5.8), we obtain

$$
\left|u^{\sigma}(x, t)-u_{0}(x)\right| \leq \omega_{\sigma, R}(t) \text { for } x \in B(0, R), t \in[0, \infty)
$$

for each $R>0$, where

$$
\begin{aligned}
\omega_{\sigma, R}(t) & =\sup _{x \in B(0, R)} \inf _{\epsilon \in(0,1)}\left\{M \epsilon+t\left[\frac{M n \sigma}{\epsilon}+M \alpha(\epsilon+|x|)+C_{M}+|f(x)|\right]\right\} \\
& =\sup _{x \in B(0, R)}\left\{2 M \sqrt{\operatorname{tn} \sigma(1+\alpha t)}+t\left[M \alpha|x|+C_{M}+|f(x)|\right]\right\} .
\end{aligned}
$$

Regarding any fixed $s \geq 0$ as the initial time and using (2.8) and the same construction of viscosity super- and sub-solutions as $U^{\sigma}$ and $V^{\sigma}$, we conclude (2.9).

\section{References}

[1] Alvarez, O., Lasry, J.-M., Lions, P.L.: Convex viscosity solutions and state constraints. J. Math. Pures Appl. 76, 265-288 (1997)

[2] Barles, G., Souganidis, P.E.: On the large time behavior of solutions of HamiltonJacobi equations. SIAM J. Math. Anal. 31, 925-939 (2000)

[3] Barles, G., Souganidis, P.E.: Some counterexamples on the asymptotic behavior of the solutions of Hamilton-Jacobi equations. C.R. Acad. Sci. Paris 330, 963-968 (2000)

[4] Barles, G., Souganidis, P.E.: Space-time periodic solutions and long-time behavior of solutions to quasi-linear parabolic equations. SIAM J. Math. Anal. 32, 1311-1323 (2001)

[5] Crandall, M.G., Ishii, H., Lions, P.L.: User's guide to viscosity solutions of second order partial differential equations. Bull. Am. Math. Soc. 27, 1-67 (1992)

[6] Davini, A., Siconolfi, A.: A generalized dynamical approach to the large time behavior of Hamilton-Jacobi equations. SIAM J. Math. Anal. 38, 478-502 (2006) (electronic)

[7] Fathi, A.: Sur la convergence du semigroup de Lax-Oleinik. C.R. Acad. Sci. Paris Sér. I Math. 327, 267-270 (1998)

[8] Friedman, A.: Partial Differential Equations of Parabolic Type. PrenticeHall, Englewood Cliffs (1964)

[9] Fujita, Y., Ishii, H., Loreti, P.: Asymptotic solutions of viscous HamiltonJacobi equations with Ornstein-Uhlenbeck operator. Commun. Partial Differ. Equ. 31, 827-848 (2006) 
[10] Fujita, Y., Ishii, H., Loreti, P.: Asymptotic solutions of Hamilton-Jacobi equations in Euclidean $n$ space. Indiana Univ. Math. J. 55, 1671-1700 (2006)

[11] Fujita, Y., Uchiyama, K.: Asymptotic solutions with slow convergence rate of Hamilton-Jacobi equations in Euclidean $n$ space. Differ. Integr. Equ. 20, 1185-1200 (2007)

[12] Ishii, H.: Asymptotic solutions for large time of Hamilton-Jacobi equations in Euclidean $n$ space. Ann. de l'IHP-Analyse Non Lineaire 25, 231-266 (2008)

[13] Ishii, H.: Personal communications

[14] Namah, G., Roquejoffre, J.-M.: Remarks on the long time behaviour of the solution of Hamilton-Jacobi equations. Commun. Partial Differ. Equ. 24, 883-893 (1999)

[15] Rockafellar, R.T.: Convex Analysis, Princeton Mathematical Series, vol. 28. Princeton University Press, Princeton (1970)

[16] Roquejoffre, J.-M.: Comportement asymptotique des solutions d'équations de Hamilton-Jacobi monodimensionnelles. C.R. Acad. Sci. Paris t. 326(Serie I), 185-189 (1998)

[17] Roquejoffre, J.-M.: Convergence to steady states or periodic solutions in a class of Hamilton-Jacobi equations. J. Math. Pures Appl. 80, 85-104 (2001)

Y. Fujita

Department of Mathematics, University of Toyama,

Toyama 930-8555, Japan

e-mail: yfujita@sci.u-toyama.ac.jp

P. Loreti

Dipartimento di Metodi e Modelli Matematici per le Scienze Applicate, Sapienza Universita' di Roma, Rome, Italy

e-mail: loreti@dmmm.uniroma1.it

Received: 29 September 2008.

Accepted: 24 May 2009. 